\title{
Geographic Process Modeling Based on Geographic Ontology
}

https://doi.org/10.1515/geo-2018-0061

Received April 10, 2018; accepted September 25, 2018

\begin{abstract}
Considerable attention has been paid to geographic process-based studies in geographic information science research. Finding appropriate methods to express geographic processes is challenging, and working to reveal the dynamic evolution and underlying mechanisms behind these processes is worthwhile. This research proposes a process-centric ontology model that describes the geographical environment from three perspectives, namely, geographic scenes, geographic processes and geographic elements. These three aspects are combined to represent the dynamic changes of geographic phenomena. This research proposes a framework and constructs ten sub-ontologies. These sub-ontologies include the Element ontology, Scene ontology, and Process ontology. A soil erosion process is then selected to demonstrate the applicability of this framework. The entire process is divided into three sub-processes (detachment, transport and deposition), and each sub-process is described by identifying when and where the process occurred, the elements and their reactions, and the changes in the geographic scene. Different relationships among elements, scenes and processes are defined to explain how and why soil erosion occurred. This proposed approach can reveal the underlying mechanisms of geographic scenes, can be used to explore the occurrence and causes of geographic processes and support the complex representation of geographic elements.
\end{abstract}

Keywords: Geographic Process Modeling; Geographic Ontology; Geographic Scene; Soil Erosion Modeling

\footnotetext{
Yuwei Cao, Yi Huang: Key Laboratory of Virtual Geographic Environment (Nanjing Normal University), Ministry of Education, Nanjing, 210023, China

State Key Laboratory Cultivation Base of Geographical Environment Evolution (Jiangsu Province), Nanjing, 210023, China Jiangsu Center for Collaborative Innovation in Geographical Information Resource Development and Application, Nanjing, 210023, China

Jing Chen: Department of Art and Design, Nanjing Audit University Jin Shen College, Nan Jing ,China
}

\section{Introduction}

Everything in the physical world is a process [1]. GIS (geographic information system) technology is currently transitioning from a traditional emphasis on the expression of geographical information to a focus on the process of changes in information [2]. The modeling of geographic processes is the basis for analyzing spatial-temporal phenomena and extracting related high-level knowledge. The expression and analysis of geographic processes has become the focus of GIS research. Geographic processes are used to describe how geographic elements evolve and the interactions between these elements [3]. The expression and analysis of geographic processes should not only include basic geographical information, such as location (where), time (when) and geographic elements (what), but also elaborate on the evolution of geographic elements during geographic processes (how), the process of this evolution (why) and deeper spatial-temporal semantic problems [4]. Expressing the evolution of geographic processes expresses how geographic elements and geographic scenes change through time and space during geographic processes. The interaction among geographic elements reflects the internal mechanisms of geographic processes and answers the important question of why geographic processes occur.

The modeling and expression of geographic processes can be constructed as a modeling framework based on geographic elements that describe changes in geographic elements at specific times and locations, depict the states of geographic elements, and actualize local dynamic expression. However, the geographic element-centric mod-

\footnotetext{
*Corresponding Author: YeHua Sheng: Key Laboratory of Virtual Geographic Environment (Nanjing Normal University), Ministry of Education, Nanjing, 210023, China

State Key Laboratory Cultivation Base of Geographical Environment Evolution (Jiangsu Province), Nanjing, 210023, China Jiangsu Center for Collaborative Innovation in Geographical Information Resource Development and Application, Nanjing, 210023, China

E-mail: shengyehua163@163.com; Tel.: +86-137-7060-0707
} 
eling method lacks the expression of complex spatialtemporal relations and semantic relations among geographic elements $[5,6]$. This method can only partially answer the questions when, where and what and cannot express how geographic elements evolve at a particular time. This method also cannot reveal the mechanisms underlying geographic phenomena or internal geographic processes, nor can it answer the questions how and why. Researchers recommend this modeling method based on geographic events $[7,8]$. This method can be used to describe the evolution of geographic elements, express these processes through a chain of events, employ events as historical snapshots of objects, emphasize the importance of events, and describe the what and where questions regarding geographic elements. However, the event-centric modeling method emphasizes the results of these events and focuses on the state of a geographic element at a certain point in time but cannot explore how and why these events occur and lacks expression of the interaction mechanisms of geographic elements. Thus, Xue [9] and Xie [10] have proposed a process-centric geographic modeling method to express the internal mechanisms of events and establish relationships, such as process-event-elements. This method also describes the relations among events and elements of the geographic process. In addition, this method expresses the evolution of the spatial relationships of geographic elements during geographic processes $[7,11]$.

Ontology is a conceptual, formal, explicit, and shared specification [12]. It is a formal description of the conceptual model, whereas unified modeling language (UML) is a graphical representation of the object oriented model; both are embodiments of information. The difference is that ontology can express more information that is understandable to both humans and computers. Ontology is the precise definition of the concept of knowledge and the relationship among concepts. Ontology can clearly express concepts and implied knowledge and aid in the formation of a knowledge network by mapping different concepts and the extension and association of concepts. This approach objectively represents the conceptual structure of the geographic world [13]. This approach helps one understand specialized knowledge and allows people to reach a common understanding along with a commonly accepted recognition and definition of a concept. Ontology is popular in computer science and is related to geography [14]. Geographic ontology is a domain ontology and a clear and formalized specified description of the shared conceptual model in GIS [15]. Geographic ontology is an extension of ontology to geospatial information [16] and belongs to a subset of ontology. With the rapid development of geospatial technology, research on geo- graphic ontology is increasingly important. The National Institute of Electrical and Electronics Engineers (IEEE) established a standard upper ontology working group to develop Suggested Upper Merged Ontology (SUMO) [17]. The U.S. Department of Defense collected the "2002 CIA's basic national conditions"-information to develop a set of traffic domain ontologies [18]. Environment Ontology (ENVO) consists of classes (terms) that refer to key environment types that may be used to facilitate the retrieval and integration of a broad range of biological and geological data [19]. To promote interoperability with established geographic resources, many of ENVO's geographic feature classes have synonyms that reference terms in geographic resources, such as the USGS vocabularies, Alexandria Digital Library's [20], the GeoNames geographical database's feature classes, and the earthrealm ontologies of Semantic Web for Earth and Environmental Terminology (SWEET)[21]. SWEET ontologies are noteworthy because SWEET is the largest geoscience data and terminology research project and is supported by NASA [21]. SWEET focuses on the expression of common concepts in the field of geographical information and disregards the most important time and spatial relationships in geography, which hinder the expression of the evolution and interaction of geographic elements. The classification of geographic processes is detailed. However, these classifications are not related to other ontologies; thus, the expression of the relation among geographic fields, life entities, non-living entities, time and space in the process of ontology is difficult.

Geographic ontology provides a possible method of clearly expressing geographic processes. Using geographic ontology was proposed as the basic tool to express concepts such as geographic processes, geographic events, and geographic phenomena $[6,22,23]$. The research on geographic ontology previously primarily focused on ontological theory [17, 24, 25], knowledge engineering [26-28], information integration [29-32] and information retrieval $[33,34]$. For the expression of geographic processes, Grenon and Smith[6] proposed SNAP to describe an instantaneous process and SPAN ontology to describe a continuous process. To characterize different types of geographic processes, the model divides the geographic process into an instantaneous process and a continuous process. The model then expands the spatial relations on the upper ontology of BFO [17] to provide spatial reasoning capability. LemosDias [22] constructs the bottom-up hierarchy of processes, sub-processes, events and operations; expresses the interactions of geographic elements during the geographic process; and then constructs events and processes to fully elaborate the geographic process. The process-oriented geographic ontol- 
ogy establishes a spatial-temporal ontology and uses the evolution of time and space to express the basic content of geographic process ontology and the basic function of the geographic process. This approach accurately depicts the content of the geographic process. This method can also answer when and where the process occurs and can describe the appearance, disappearance and evolution of its elements and how processes interact with each other.

The problems with these ontology-oriented studies can be summarized as follows: (1) they ignore the integrity of the geographic process. The studies of geographic processes tend to focus on the evolution and interaction of geographic elements. This research focuses on the effects of element to element and element to process but lacks the efforts of element and process to the entire geo-scene and the interrelationship between geo-scenes, which disregards the role of elements or processes in the geographic scene and the macroscopes interaction and evolution in geo-scenes. (2) These studies cannot characterize the relationship among geographic processes, geographic elements and geographic scenes. The expression of geographic processes in the available model primarily focuses on the process of the changes in a single geographic element. This approach hinders the characterization of multielement and multi-level complex relationships in the geographic scene. (3) These studies do not clarify the semantic relations of these processes. The expressions of ontology in previous studies could describe only the participants of these processes. These works were incapable of expressing these processes' deeper semantic relations. These relations include the role of participants during this process and the functions and abilities of the participants. (4) These studies fail to clearly express the space-time relationship. There are few studies of spatial relations and time relations in geographic ontology. Even if a few of these studies involve spatiotemporal ontology, the processes can be described based only on a single part and cannot describe the complicated spatiotemporal relations of ontology.

In view of the above problems, this paper constructs an ontological model that focuses on the ontology of the geographic process, expresses its complex relations, and reveals its internal mechanism. This paper proposes the 'geographic scene ontology' to describe the total changes in the geographic scene during the geographic process, resolve the lack of integrity in the expression of the geographic process, employ the characteristics of the ontology to overcome the semantic ambiguity in the expression of the geographic process, and introduce time ontology and spatiotemporal ontology to describe the evolution of geographic processes. This method can clearly and completely express the semantics, semantic relations, spatiotemporal elements, evolutionary processes, and internal mechanisms of geographic processes and can answer the questions when, where, what, why, and how.

\section{Materials and Methods}

\subsection{Geographic Process of Expression}

To represent the ontology of geographic processes, the concepts of the geographic scene, geographic element and geographic process that are closely related to the geographic process should be analyzed, and their relations should be described.

\subsubsection{Definition and Relationship of Concepts}

A geographic scene can be defined as a specific regional synthesis that comprises both human factors and natural factors and their mutual relationships and interactions. A geographic scene has a specific structure and functions and is characterized by comprehensiveness (involves all types of geographic factors, such as water, soil, the atmosphere, biology, and human activities), multi-hierarchy (can support the description of a region at different hierarchical levels) and multi-dimensionality (two-dimensional (2D), three-dimensional (3D), and the temporal dimension) [35].

Geographic elements are geographical objects with definite locations and morphological characteristics in geographical space or geographic phenomena. The connotation of geographic elements includes semantic descriptions (semantics is the meaning of the descriptions of scenes and inner geographic elements), spatial positions, geometrical forms, evolution processes, attributes (a geographic attribute reflects the characteristics of a geographic element and is an important basis of element classification) and element relations.

A geographic process is an action that causes the geographical entity to change and emphasizes the underlying mechanism of the geographic process [36]. Yuan [37] suggests that a geographic process is a series of orderly dynamic correlation states that show how geographic elements evolve in time and space; this definition emphasizes the evolution of the geographic elements in a process. This paper indicates that the geographic process is the combination of the two approaches, that is, a geographic process is the evolution and interaction of geographic elements 
and a geographic scene in time and space. A geographic process can include several sub-processes. This paper uses the terms "state" and "action" to describe a geographic process. The term "State" is used to express changes in the scene and the elements in a geographic process, and the term "action" is used to express the interaction among the elements. Each geographic process can be subdivided into several distinct sub-processes. For example, the process of sediment yield in the basin includes the sub-processes of rainfall, surface runoff and soil erosion.

In the past, the classification of geographic processes has mainly divided geographic processes into the instantaneous processes (e.g., explosion) and continuous processes (e.g., rainfall) according to the duration of the process $[7,29]$. The evolution of the geographic process can be described by time points and time segments. However, this method may vary with temporal resolution, resulting in a different division of the process. Therefore, starting from the results, this paper divides geographic processes into two types: the first type of process changes the properties (e.g., geometric and semantic properties) of a geographic scene through changes in geographic elements. The second type of process changes the geographic elements in the scene due to the changes in the total scene.

Geographic elements are the smallest units of change in a geographic process. Based on the connotation of different geographic elements in the process, this study elaborates on the spatial structure and form, attributes, semantics and spatiotemporal relations of these changes. The types of changes to geographic elements are classified according to the number of elements that participate in the process (Table 1). Spatial structure and form are the spatial elements and geometrical forms of geographic elements that describe the shape and spatial position of the elements. Attributes are the attributes of geographic elements; they describe the intrinsic properties of geographic elements. Semantics are the semantic elements of geographic elements; they describe the meaning of external endowed elements. A semantic relation refers to the relations that describe geographic elements; they comprise the relationship between the elements in the geographic scene and other geographic elements. A spatiotemporal relationship refers to the characteristics of the evolution of geographic elements and describes the spatial and temporal relationship between the geographic elements and other elements.

The three concepts of geographic processes, geographic scenes and geographic elements are coupling relations. Geographic scenes include geographic elements and relations among geographic elements. Geographic processes occur in geographic scenes. Geographic elements participate in geographic processes. Geographic elements and geographic scenes change with time in geographic processes. In a geographic process, geographic elements are represented by state and action. Geographic elements have different types of changes in a geographic process; these types of changes are related to other involved elements and the process. These three concepts have unique inner associations; for instance, the time relations among different geographic processes, and the spatial relations among different geographic elements or scenes.

\subsubsection{Modeling Relationships Among Geographic Processes, Geographic Scenes, Geographic Elements}

UML is used to express the geographic processes, geographic scenes and the relationship among geographic elements. Among them, the inheritance relation in UML represents the parent-child relationship; the aggregated relation represents the contain and part-of relations; semantic relationships and space-time relationships are represented by the association relationships; and association relationships are described in the following ontology model. In the diagram in Figure 1, the geographic scene consists of geographic elements and is associated with a geographic process and the geographic element is associated with the geographic process. Association relations include semantic relations, space-time relations and interaction relations among elements (physical, chemical, biological and geographic function). Association relations describe the processes, elements, scenes and the relationship between these three items to express the evolution of elements and scenes in the geographic process. The association relation of an element to an element and an element to scenes is used to represent the inherent mechanism of a geographic process. These relations work in concert and clearly show the integrity, evolution and internal mechanisms of the geographic process.

\subsection{Geographic Process-Centric Ontology Model}

\subsubsection{Ontology Model Classification and Hierarchy}

To clearly express the contents of geographic processes, geographic elements and geographic scenes, this paper has proposed ten basic ontologies: geographic scene ontology, geographic element ontology, process ontology, time ontology, temporal relation ontology, space ontology, 
Table 1: Types of Geographic Element Changes in Geographic Processes

\begin{tabular}{lll}
\hline PROPERTIES & SINGLE ELEMENT & MULTIPLE ELEMENTS \\
\hline \multirow{3}{*}{ Spatial Structure and Form } & $\begin{array}{l}\text { Appearance, Disappearance, } \\
\text { Stability, Expansion, } \\
\text { Contraction, Deformation, } \\
\text { Replacement, Rotation, etc. }\end{array}$ & $\begin{array}{l}\text { Appearance, Disappearance, } \\
\text { Stability, Expansion, } \\
\text { Contraction, Deformation, } \\
\text { Replacement, Rotation, Union, } \\
\text { Split, etc. }\end{array}$ \\
\hline \multirow{2}{*}{ Attributes } & $\begin{array}{l}\text { Physical attributes, Chemistry } \\
\text { attributes, Biological } \\
\text { attributes, etc. }\end{array}$ & $\begin{array}{l}\text { Physical attributes, Chemistry } \\
\text { attributes, Biological } \\
\text { attributes, etc. }\end{array}$ \\
\hline Semantic & Quantity, Role, Function, & Quantity, Role, Function, \\
& Power, etc. & Power, etc. \\
\hline Semantic Relations & & $\begin{array}{l}\text { Instant-of, submission-of, } \\
\text { part-of, etc. }\end{array}$ \\
\hline Temporal Relations & & $\begin{array}{l}\text { Time sequence relation, Time } \\
\text { topological relation }\end{array}$ \\
\hline Spatial Relations & & $\begin{array}{l}\text { Directional relation, Distance } \\
\text { relation, Topological relation }\end{array}$ \\
\hline
\end{tabular}

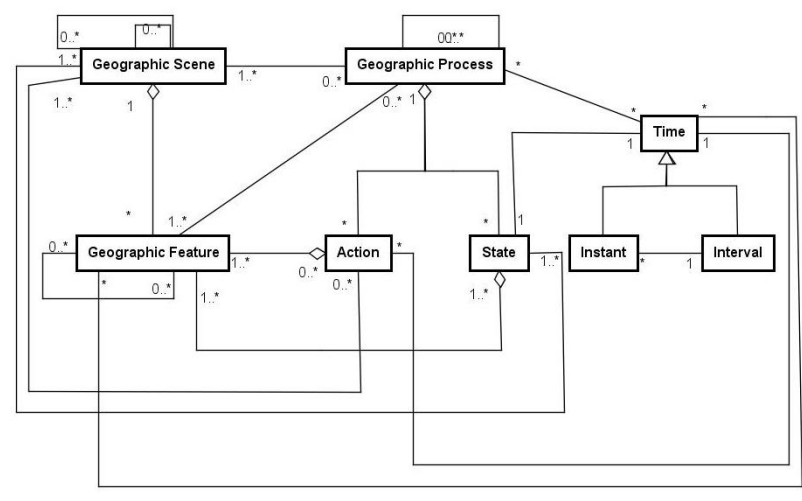

Figure 1: Relations among Geographic Processes, Geographic Scenes, and Geographic Elements

spatial relation ontology, representation ontology, substance ontology, and operation ontology (Table 2). Geographic scene ontology is used to represent a collection of geographic scenes. According to different analysis needs, geographic scenes can be divided into different sub-scenes. For example, a geographic scene can be divided into a land scene, a sea scene and an event scene, and then, the land scenes are subdivided into a natural scene, a business scene, and an artificial scene. The natural scene includes a mountain scene, a desert scene, and a polar scene.

\subsubsection{Elements' Relationship Expression in Ontologies}

The interactions of geographic processes include a variety of types of semantic relationships, such as elements to scenes, elements to elements, processes to scenes and elements to processes. These relationships need to be able to describe the geographic process completely. The relationships can be divided into semantic relations, space-time relations and the interactions between elements (physical, chemical, biological, and geographical) (Table 3). These relations are used to link the classes in ontology and classes out of ontology to associate the geographic processes with geographic scenes and geographic elements and form a complete model.

Therefore, there we can derive two conclusions. First, each geographic process has many associated ontologies. For example, almost all the ontologies are related to the sediment production process. Sediment belongs to the Substance ontology, the basin belongs to the Geographic Element ontology, and the geographic elements included in the process are associated with the Geographic Scene ontology. Second, the geographic process includes both the internal relationship and the relationship between ontologies. Both relationships have semantic relations, space-time relations and interactions. The relationships between ontologies, such as the relationship among geographic elements and geographic processes, comprise a participant relationship that belongs to semantic relationships. For example, SedimentOfBasin hasParticipantOf Sediment indicates that sediment is a participant in the sediment production process; SedimentOfBasin belongs 
Table 2: Concepts of Ontologies

\begin{tabular}{|c|c|c|}
\hline ONTOLOGIES & DESCRIPTION & CLASSS \\
\hline $\begin{array}{l}\text { Geographic Scene } \\
\text { Ontology }\end{array}$ & $\begin{array}{l}\text { Hierarchical geographic scene } \\
\text { classification. The formation of a specific } \\
\text { structure and function of the } \\
\text { geographical complex - geographic } \\
\text { scene ontology }\end{array}$ & $\begin{array}{l}\text { Land scene (natural scene } \\
\text { (woodland, desert...), } \\
\text { business scene, artificial } \\
\text { scene), sea scene } \\
\text { (offshore, far sea), event } \\
\text { scene (disaster scene) }\end{array}$ \\
\hline $\begin{array}{l}\text { Geographic Element } \\
\text { Ontology }\end{array}$ & $\begin{array}{l}\text { With reference to GBT, the geographic } \\
\text { elements are classified into different } \\
\text { types and hierarchical levels to form the } \\
\text { geographic element ontology }\end{array}$ & $\begin{array}{l}\text { Water system, residential } \\
\text { area and facilities, } \\
\text { transportation, pipeline, } \\
\text { boundary, landform, soil, } \\
\text { vegetation }\end{array}$ \\
\hline Process Ontology & $\begin{array}{l}\text { A collection of various processes, such } \\
\text { as physical processes, process } \\
\text { ontologies and other ontologies, are } \\
\text { linked, such as the processes that occur } \\
\text { in the scene and the elements evolved in } \\
\text { these processes }\end{array}$ & $\begin{array}{l}\text { Physical processes } \\
\text { (adsorption), chemical } \\
\text { processes (decomposition), } \\
\text { geographic processes (karst } \\
\text { processes), biological } \\
\text { processes (photosynthesis), } \\
\text { complex processes }\end{array}$ \\
\hline Time Ontology & $\begin{array}{l}\text { A collection of time-related concepts and } \\
\text { a complete time representation } \\
\text { associated with the representation } \\
\text { ontology }\end{array}$ & $\begin{array}{l}\text { Time coordinate sys- } \\
\text { tem, time point, time period }\end{array}$ \\
\hline $\begin{array}{l}\text { Temporal Relation } \\
\text { Ontology }\end{array}$ & The relation collection between time & $\begin{array}{l}\text { Time sequence relation, } \\
\text { time topology relations }\end{array}$ \\
\hline Space Ontology & $\begin{array}{l}\text { The collection of space-related concepts, } \\
\text { such as spatial geometry, spatial } \\
\text { coordinates, spatial extent, combined } \\
\text { with the units representing the ontology, } \\
\text { represents the complete space }\end{array}$ & $\begin{array}{l}\text { Geometry, spatial } \\
\text { coordinate system, } \\
\text { coordinates, spatial } \\
\text { position, space range }\end{array}$ \\
\hline Spatial Relation Ontology & $\begin{array}{l}\text { The collection of spatial relations among } \\
\text { elements }\end{array}$ & $\begin{array}{l}\text { Distance relation, } \\
\text { directional relation, } \\
\text { topological relation }\end{array}$ \\
\hline Representation Ontology & $\begin{array}{l}\text { The ontology used to denote the } \\
\text { properties, units, values, states, etc., of } \\
\text { other ontologies }\end{array}$ & $\begin{array}{l}\text { Unit, attribute (quality, role, } \\
\text { function, capability), value, } \\
\text { conversion factor, status } \\
\text { (element status and scene } \\
\text { status) }\end{array}$ \\
\hline Substance Ontology & $\begin{array}{l}\text { Used to represent a component of a } \\
\text { element, such as a physical part }\end{array}$ & $\begin{array}{l}\text { Physical composition, } \\
\text { chemical composition, } \\
\text { biological composition }\end{array}$ \\
\hline Operation Ontology & $\begin{array}{l}\text { Used to represent the interaction of } \\
\text { elements }\end{array}$ & $\begin{array}{l}\text { Physical effects (gravity, } \\
\text { friction), chemical effects, } \\
\text { biological effects, } \\
\text { geographic effects (wind } \\
\text { erosion, etc.) }\end{array}$ \\
\hline
\end{tabular}

to the Geographic Element ontology; Sediment belongs to Geographic Process ontology; and hasParticipantOf is an object property. The status of geographic elements and geographic scenes changes over time. This change is related to geographic elements by the state property in the Semantic ontology. For example, the state of the soil's separa- tion is expressed as Soil hasStateOf Detached, which indicates that the soil has a detached state. The locational relationship among the geographic elements is a spatial relation. For example, the relationship between soil and runoff is an adjacent relation, and the Spatial Relation ontology represents these relationships as Soil hasMeetSpa- 
Table 3: Relationship between Elements

\begin{tabular}{ll}
\hline TYPE OF RELATION & RELATION \\
\hline \multirow{3}{*}{ Semantic relationship } & $\begin{array}{l}\text { Instant-of, subsumption, } \\
\text { part-of, participation, } \\
\text { attribute-of, inherence, } \\
\text { same-as, contrast-of } \\
\text { disjoint-of }\end{array}$ \\
\hline \multirow{3}{*}{ Temporal relationship } & $\begin{array}{l}\text { Sequence relation (before, } \\
\text { after, etc.), time topological } \\
\text { relation (disjoint, meet, etc.) }\end{array}$ \\
\hline Spatial relationship & $\begin{array}{l}\text { Distance relation (near, } \\
\text { Euclidean-metric), directional } \\
\text { relation (north, west, etc.), } \\
\text { spatial topological relation } \\
\text { (disjoint, meet, etc.) }\end{array}$ \\
\hline Interaction relationship & $\begin{array}{l}\text { Physical-operation, } \\
\text { chemical-operation, } \\
\text { biological-operation, } \\
\text { geographic-operation }\end{array}$ \\
\hline
\end{tabular}

tialRelationWith RunOff. Similarly, there is a time relationship between processes; for example, the soil separation process takes place earlier than the runoff process, and through the Time Relation ontology, this relation is express as SoilDetachedProcess hasBeforeTimeRelationWith SoilTransportProcess, and SoilDetachedProcess and SoilTransportProcess belong to the process ontology, while hasBeforeTimeRelationWith is the object property. The relationships between geographic elements have an interaction relationship, which belongs to the Operation ontology. For example, the interactions between surface runoff and soil include erosion, detach, decomposition and other physical effects, and through the operation ontology, these interactions are represented as Runoff Detach Sediment. The internal ontology relationship is much more than the relationship between ontologies and includes the inheritance relationship, sibling relationship and instance relationship. In the inheritance relationship, the son inherits the property of the father, and there are common attributes inherited from the father, but there are also differences. The instance relationship is equivalent to the relationship between the class and the instance.

The expressions of relations are mainly divided into the expression of internal relationships and the express of relationships between ontologies. Both relationships can be connected by object properties and data properties; for example, for soil elements and tree elements, the

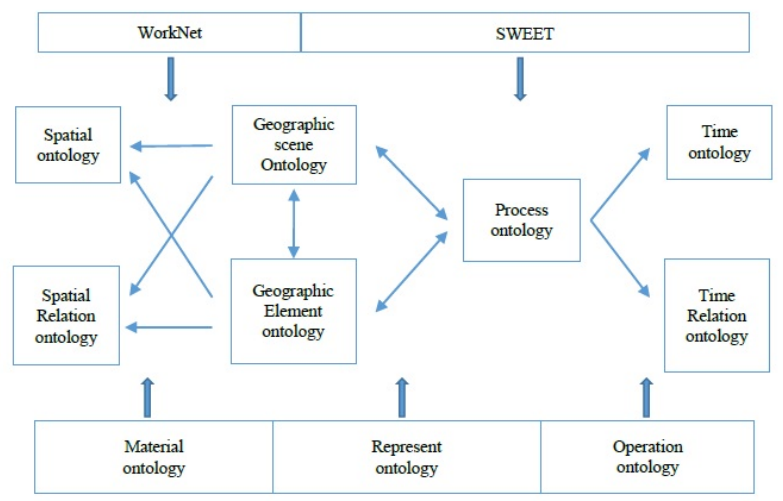

Figure 2: Geographic Ontology Model Diagram

roots of the tree are in the soil, and the stems and leaves are in the air. Therefore, there is a relationship between the tree instance and the soil instance, and the relationship between the two is described as Soil hasDisjointRelationshipwith Plant. A property such as geographic coordinates described by a specific value has the data property hasCoordinate, which can be described as follows: Coordinate1 hasCoordinate 23. In addition, the expression of other internal relationships in the ontology can be directly expressed by constructing the nested relation between the ontology and the objects in this ontology.

\subsection{Ontology Model}

Through the above analysis of the components of geographic ontology, the relations among ontologies and the expression of relations, this paper focus on Process Ontology, according to the association relationship between Scene ontology, Element ontology, Time ontology, Temporal Relation ontology and Process ontology and the association relationship among Spatial Relations ontology, Space ontology and Element ontology, and Scene ontology. These seven ontologies represent the center of the network ontology model. Then, with reference to ontologies defined by SWEET and SUMO, several common concepts in the geographical domain are imported. The last step is to construct the Substance ontology, Representation ontology and Operation ontology to support the expression of the geographic process. For example, the Substance ontology is taken as the basic component of the element ontology; the unit object in the Represent ontology is taken as the unit of some properties of the instances of the Geographic Element ontology; and the Operation ontology is taken as the basis for describing the interaction among elements. 
The composition of the building ontology can be cumbersome and sometimes overconsumed. One possible solution to this problem is to reuse the ontology that has been created by others [38]. Therefore, in the construction of the geographic process ontology, the concepts and classes in the WordNet and SWEET ontology are cited. WordNet is a large lexical database of English. Nouns, verbs, adjectives and adverbs are grouped into sets of cognitive synonyms (synsets); each set expresses a distinct concept. Synsets are interlinked by conceptual-semantic and lexical relations. Thus, the common concepts or terms, such as time, space, and human, can be referenced from WordNet. Some geographic and process concepts, such as location, coordinate, and physical process, can be cited from the SWEET ontology. We can reference these classes by the identifiers of these classes in other OWL ontologies, such as SWEET or SUMO. For example, for the soil class, we can use the relative identifier \#Soil. For instance, since other documents may also need to reference this class, the most reasonable method is to provide a namespace and entity definition, including the definition document of this class as the definition source, as shown in Figure 2. Then, the Spatial ontology, Spatial Relation ontology, Geographic Scene ontology, Geographic Element ontology, Process ontology, Time ontology, and Temporal Relationship ontology are taken as the core ontologies. This paper uses the relationships among the ontologies themselves to express the evolution and internal mechanisms of geographic processes, whereas the qualities and attributes of the elements are completed using three basic supporting ontologies. Through the interaction of these ontologies, we can complete the expression of the geographic process ontology shown in Figure 4 and answer how and why processes occur.

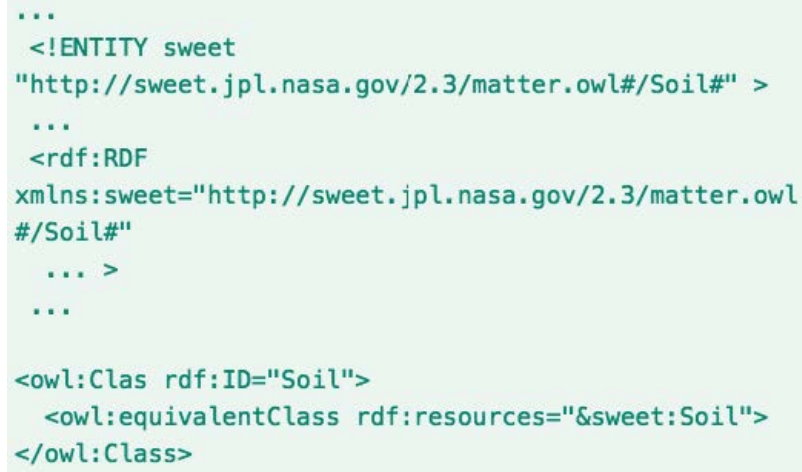

Figure 3: Method to reference other ontologies

\subsection{Formalized}

After the ontology classification, attributes, and relationships are established, and the ontology model is constructed, we need to formalize and express the ontology through the formal ontology description language and employ the ontology construction tool to emphasize the concepts, relationships, and attributes of the ontology model. Formal coding with examples and other content is needed to enable formal use of the conceptual model to facilitate computer understanding. Many existing geographic ontology expression languages, such as $\mathrm{OWL}, \mathrm{RDF} / \mathrm{RDF}(\mathrm{s})$, XML/XML(s), DAML, OIL, KIF, and Ontolingua, are recommended. OWL is the $\mathrm{W} 3 \mathrm{C}$ recommended ontology description language, from the early ontology language OI, DAML and DAML+OIL. Compared with ontology languages such as XML and RDF, vocabulary with more descriptions of classes and attributes is better expressed in terms of expressing lexical meaning and semantics. For example, the following concepts and attribute constraints can be established through OWL: The hierarchical structure of the concepts, such as the inheritance relationship, can be implemented by Subclass, SubPropertyOf; the logical combination relationship among concepts can be realized by IntersectionOf and UnionOf; and the attribute constraint is implemented by Range and Domain. For some parts of OWL, some semantic formal expressions that are required in process ontology modeling, such as roles and functions, need to be manually added. For instance, we construct a Role class and set its restriction and the object property to connect two elements.

\section{Case Study}

\subsection{Soil Erosion Process Ontology Model}

To provide an understanding of the connotation of the above geographic process and the ontology expression method, the process of soil erosion is taken as an example. This paper depicts the temporal-spatio process of soil erosion, the scene of soil erosion, the elements involved in the process of soil erosion and their relationships to express the evolution of this geographic process and the mechanism of internal action.

The process of soil erosion is essentially the process of a change in soil spatial position under the action of natural external forces (Xu, 2001). Changes in matter and energy are fundamental drivers throughout the entire process. As a result, the soil is detached, transported and deposited. 


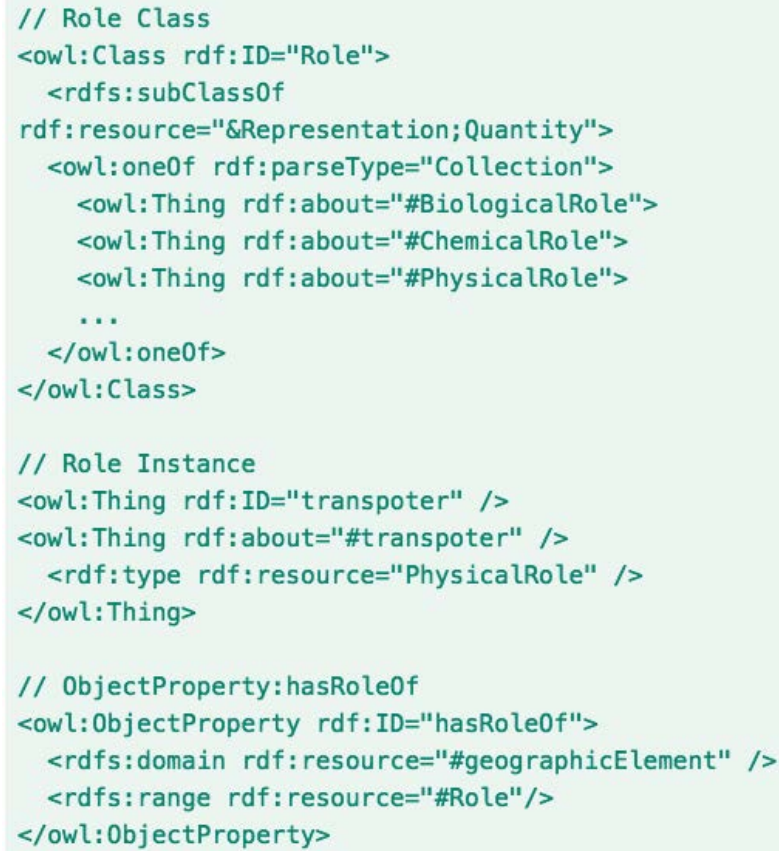

Figure 4: Definition of Role Class

The soil detachment process, transport process and deposition process are sub-processes of the soil erosion process. In this experiment, the mechanisms and evolution processes of these three sub-processes are analyzed and an ontology concept modeling approach is proposed.

Soil erosion occurs because rainfall and runoff have energy. The soil erosion process is essentially a process of energy consumption. Soil detachment refers to the process in which soil particles leave the original position when the runoff forces acting on the soil particles are greater than the resistance between the soil particles. Sediment transport means that the detached soil particles are carried away by runoff. Sediment deposition occurs when the amount of sediment in the upper stream is larger than the transport capacity of runoff. These three processes are mutually influential, mutually restrictive and interrelated. The amount of soil detached by runoff is also affected by runoff transport capacity. The rate of soil detachment by runoff is generally accepted as inversely proportional to its transport rate. If the transfer rate of runoff increases, the amount of energy used for transport increases, and the ability to detach the soil decreases. The transport rate in turn depends on the runoff transport capacity; the greater the transport capacity is, the higher the transfer rate will be. An analysis of the elements of these three processes, the inter-element interactions, and the attributes of the elements yields the results in Table 4.
According to SU's seven-step method, we construct the ontology of the soil erosion scene and enumerate the main terms in the scene in the well-defined thesaurus and ontology (WordNet, SWEET). Next, we construct the ontology class for scene classes, process classes, geographic element classes, time classes, space classes, presentation classes, and their hierarchy. After selecting classes from the list of terms, most of the remaining terms are likely to be properties of these classes. These terms include the soil's color, body, viscosity, composition and location of a soil instance. For each property in the list, we must determine to which class it belongs. These properties become slots attached to classes. Thus, the soil class will have the following slots: color, viscous, composition and location. However, the slots can have different facets, domain, and range that describe the value type, allowed values, the number of the values (cardinality), and other features of the values attained by the slot. For example, the value of a name slot (for example, "the name of a city") is one string, that is, a name is a slot with a value type string. Examples of a soil erosion scene, process, and participation factors are constructed.

\subsubsection{Construction of the entire scene}

As previously mentioned, a scene class should express the entire evolution of processes and elements. After constructing these classes, we should construct the relationships between a scene and other classes. In a soil erosion scene, we need to know the roles of processes and elements at different times, how this scene changes during processes and even the relationships among several scenes. Thus, this paper builds an instance of a soil erosion scene and its properties to express the instance. The part of the instance's ontology is shown in the following figure. For example, the ContainIn relationship between a soil instance and a soil erosion scene is observed; the process is included in the scene through object properties such as ContainIn. Similarly, the relationships between scenes and processes are constructed.

\subsubsection{Construction the evolution of soil erosion process}

To express the evolution, we need to build connections between processes and other ontologies during processes. These three sub-processes are expressed in ontologies, and the properties (attributes, time, and space) of each participant are connected through object properties and data properties. Then, these relationships, concepts and 
Table 4: Soil Erosion Process Analysis

\begin{tabular}{|c|c|c|c|}
\hline ELEMENT PROPERTIES & $\begin{array}{l}\text { SOIL DETACHMENT } \\
\text { PROCESS }\end{array}$ & $\begin{array}{l}\text { SOIL TRANSPORT } \\
\text { PROCESS }\end{array}$ & $\begin{array}{l}\text { SOIL DEPOSITION } \\
\text { PROCESS }\end{array}$ \\
\hline Participation elements & Soil, runoff & $\begin{array}{l}\text { Underlying surface, soil, } \\
\text { runoff }\end{array}$ & $\begin{array}{l}\text { Underlying surface, oil, } \\
\text { Runoff }\end{array}$ \\
\hline Attributes of elements & $\begin{array}{l}\text { 1) Soil cohesion (soil } \\
\text { composition, viscosity, } \\
\text { type, particle size, } \\
\text { quantity) } \\
\text { 2) Runoff (flow rate, flow } \\
\text { rate, runoff depth) }\end{array}$ & $\begin{array}{l}\text { 1) Soil (soil content, } \\
\text { distribution) } \\
\text { 2) Runoff (runoff depth, } \\
\text { runoff velocity, cross } \\
\text { section wide of water flow, } \\
\text { water flow structure, flow } \\
\text { resistance, water } \\
\text { temperature) } \\
\text { 3) Topography (slope, } \\
\text { slope, height difference) } \\
\text { 4) Geological conditions } \\
\text { (geological structure, } \\
\text { lithology) }\end{array}$ & $\begin{array}{l}\text { 1) Soil (soil content, } \\
\text { distribution, deposition) } \\
\text { 2) Runoff (runoff depth, } \\
\text { runoff velocity, cross } \\
\text { section of water flow, } \\
\text { water flow structure, flow } \\
\text { resistance, water } \\
\text { temperature) } \\
\text { 3) Topography (slope, } \\
\text { slope, height difference) } \\
\text { 4) Geological conditions } \\
\text { (geological structure, } \\
\text { lithology) }\end{array}$ \\
\hline Semantic of elements & $\begin{array}{l}\text { 1) Roles (decomposer, } \\
\text { decomposed) } \\
\text { 2) Function (decompose } \\
\text { soil, reduce runoff energy) }\end{array}$ & $\begin{array}{l}\text { 1) Roles (transporter, be } \\
\text { transported, bearer) } \\
\text { 2) Function } \\
\text { (transportation of soil, } \\
\text { internal friction, carrying } \\
\text { soil and runoff) }\end{array}$ & $\begin{array}{l}\text { 1) Roles (discharge, to be } \\
\text { excluded) } \\
\text { 2) Function (to discharge } \\
\text { soil runoff and reduce } \\
\text { runoff) }\end{array}$ \\
\hline
\end{tabular}

Surface runoff to overcome the friction between soil particles and soil particles, surface

Surface runoff scours the Interaction of elements soil, reducing soil viscosity to separation runoff to overcome the friction between soil particles and the surface, surface runoff to overcome friction with the underlying surface movement

1) Surface runoff speed decreases

State of elements

2) Laminar flow becomes turbulent, and 3) The soil becomes loosely ordered
1) The soil is moved

2) Soil moves with runoff

3) Runoff velocity decreases with the increase in sediment load
Runoff contains more than the amount of soil runoff sediment transport capacity, the soil can no longer be carried, the occurrence of sedimentation
1) Soil deposition

2) The remaining soil continues to be transported

3) The runoff flow rate decreases slightly, and the rate increases

The amount of sediment carried in runoff is reduced, and the runoff velocity is increased, which is between turbulence and laminar flow

1) The soil is transported, and the spatial position of the soil changes

1) Soil deposition, spatial location changes

Over time, runoff flows on the slope and the spatial location is constantly changing
2) As the soil is carried, the runoff velocity is slowed, and the spatial location is also changed
2) The runoff carrying sediment decreases, the flow rate increases, and the spatial position changes 


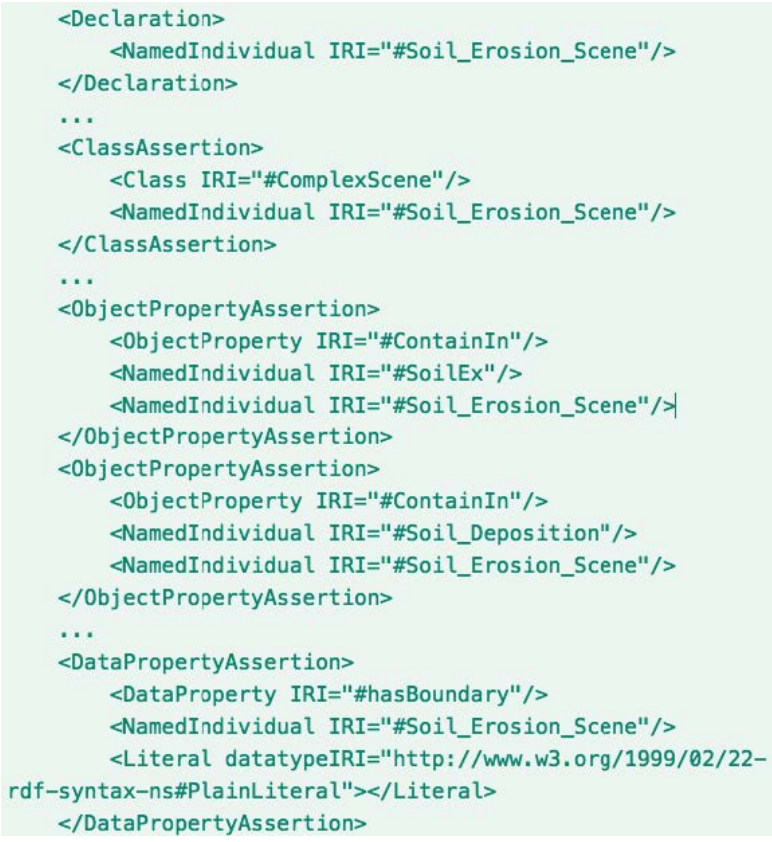

Figure 5: Definition and Relationships of Scene class

terms are implemented in the ontology modeling software Protégé. By conceptually modeling the entire process, the evolution during the entire erosion process can HasParticipantor and HasProperty, the participants in the process can connect the participants attributes. In addition, through properties such as HasState, HasSpace, HasTime, and HasSpatialRelationship, the process, state and spatial relationship of the process are connected together. The processes have a relation set to express the relations (spatial-temporal relations) with child processes. For example, the expression of the time relationship between three sub-processes is as follows: the first sub-process is soil detachment, which is loss the of adhesion between the soil, and after surface runoff erodes the soil, the soil begins to separate. Runoff begins to carry sediment, but runoff has residual energy, and this energy continues to erode the soil, thus making the soil detach further. Therefore, the time relationship between soil detachment and soil transport is as follows: soil detachment occurs before soil transport; however, in the early stages of soil transport, the two sub-processes occur simultaneously. Therefore, the temporal topological relationship is an intersection. Over time, the amount of sediment carried in runoff increases, and the amount of sediment carried exceeds the amount of sediment that can be carried by runoff, resulting in deposition. However, runoff has extra capacity to detach the soil when the deposited sediment reaches a certain amount. Therefore, the three sub-processes (detachment, transport and deposition) are cyclical in time, and among the three sub-processes exist intersection, inclusion and adjacent temporal topological relationships. Therefore, when modeling the three sub-processes with ontologies, the temporal relationships should be constructed as follows (only a part of the temporal relationships are shown)

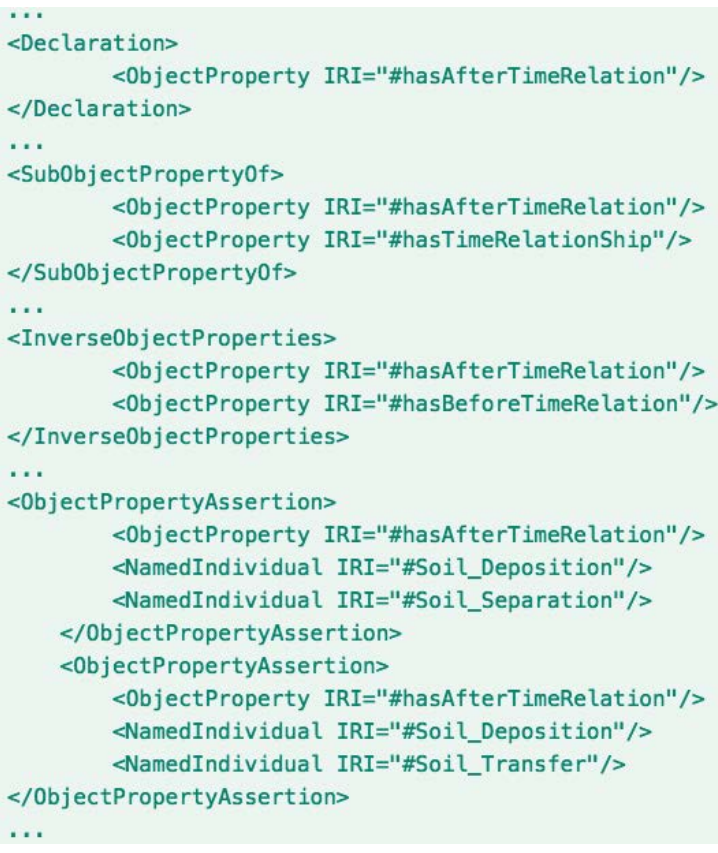

Figure 6: Part of the temporal relationships in ontology model

Similar to the construction of the temporal topological relationship among the processes, in Protégé, the relationship between instances of the soil, surface runoff, soil detachment processes, soil transport processes and time, space, spatial relations, semantics and semantic relations continued to be constructed to improve the expression of the soil erosion process. As we construct the relationship between the spatiotemporal processes and elements, we gain a clear recognition of the soil process.

\subsubsection{Express the mechanism of the soil erosion process}

In the next stage, we should consider the expression of mechanism in our ontology model, that is, properties such as actions, semantics, times are needed in this model. To express it through properties such as HasAction, HasProperty, HasRole and HasFunction, the participants in the process can connect the participants' actions, attributes, roles, and functions. In addition, through properties such as HasAction, HasState, HasSpace, HasTime, and HasSpatialRelationship, the process, action, state and spatial rela- 
tionship of the process are interconnected. The processes have a relation set to express the relations (semantics and spatial-temporal relations) with geographic elements and other processes. For example, the friction action in the Soil_Deposition process is claimed as follows, and every geographic element or process has a specific role in a particular process, time and action.

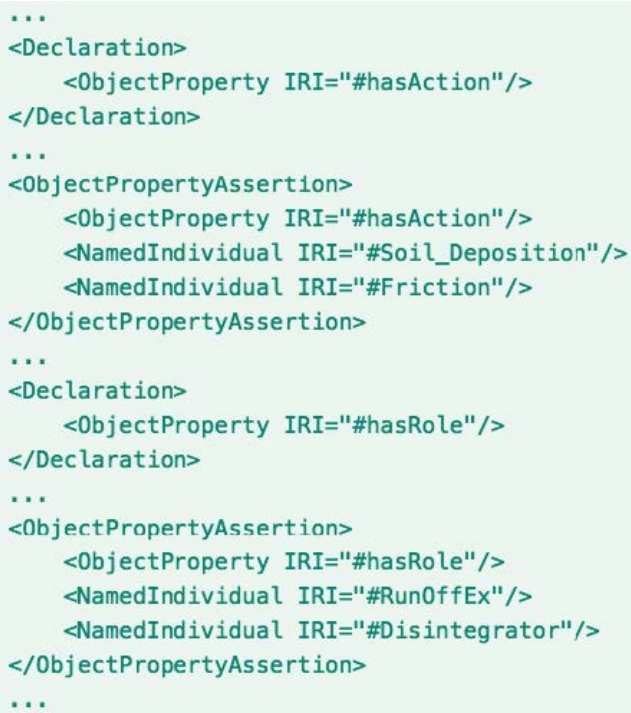

Figure 7: Parts of hasAction properties and hasRole Properties

Similar to the construction of the action, the semantic properties, such as the role, function and time properties, should be built in the ontology design. When these interactions among the geographic elements, processes and scene are constructed, the mechanism of this process should be well understood.

\subsection{Results}

After building an ontology model that can express the evolution and intrinsic mechanism of geographic processes, the results are as follows: one result is a general framework of the ontology model. Another result is the content of each ontology. Another result includes the relations with ontologies and relations among the ontologies. The ontology model (Figure 8) is composed of several ontologies described above. The various classes, object properties and data properties represent the contents and relationships of the ontologies. In the context of the soil erosion process, the associated ontologies are shown (Figure 9), and the geographic scene, geographic elements, geographic events, and composition of the geographic process and their re- lationships can be determined. Soil_Erosion_Process is the total process and contains three sub-processes and a number of actions. This process associated with other elements, such as Soil, Runoff and sandyLand, via the hasParticipant property, and events and scenes are associated with the process via the ContainIn property. Similarly, the Soil_Transport sub-process uses the hasTime and hasTimeRegion properties, which link the time and time region relations with the sub-process (Figure 10). The subprocess contains actions such as Friction, Flow, Transport and Gravity, which express interactions in the sub-process through hasAction. In addition, the hasState property associates the states of the elements and scene at different time points with the process to express the evolution of the process. Similarly, with hasParticipant, the soil instance SoilEx, the earth surface instance SandyLand, and the surface runoff instance RainOffEx are participants in the process. Then, temporal topological relations convey the temporal relationships between soil_Transport and Soil_Detachment, Soil_Deposition. The scene and process are temporally related by the ContainIn object property. Impressively, we can further express the action and state of the process. Taking the action of runoff carrying sediment transport (Figure 11) as an example, the start time, end time, and duration of the action are assigned through the following data properties: hasStartTime, hasEndTime, and hasTimeRegion, and the executors of the actions RunOffEx and SoilEx are associated with hasOperator. The predecessor (Detach) and successor (Todeposit) of the action are linked through hasPioneer and hasSuccess, indicating that the necessary prerequisite for the Transport action is to execute the Detach action and to perform the ToDeposit action. The states of the element and scene are linked to processes by the hasState property, and the actions are linked to processes by the ContainIn property. Similarly, the structure and organization of geographic scenes and geographic elements (Figure 9) are also expressed in the same manner.

\section{Conclusions}

Geographic processes can be clearly and completely expressed by constructing a process-oriented ontology model. Geographic processes, geographic scenes, geographic elements and their relationship them can be expressed. In addition, the aspects of time, space, and spatiotemporal relationships are described relative to the geographic process. The actions of geographic elements and the states of elements and scenes are also detailed 


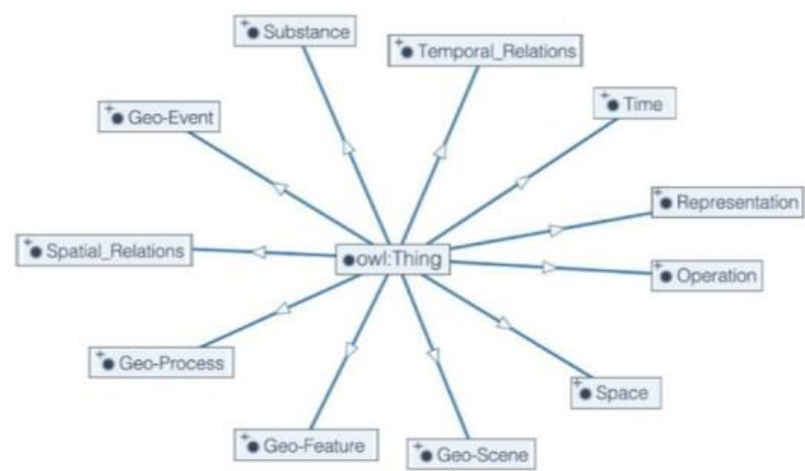

Figure 8: Framework of Ontology Model.

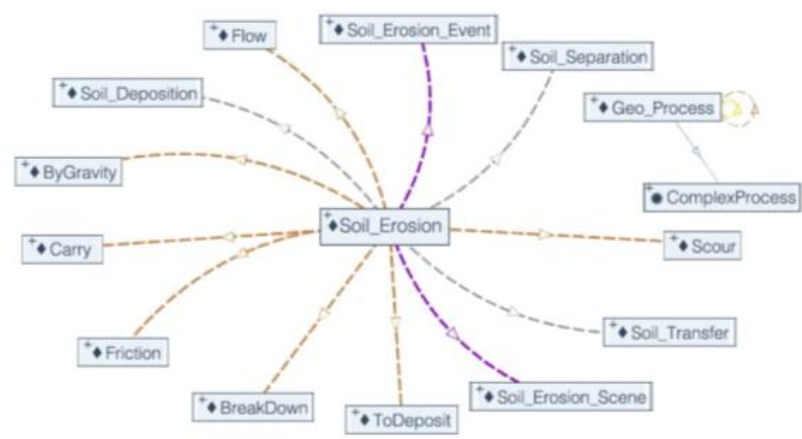

Figure 9: Soil Erosion Process Ontology.

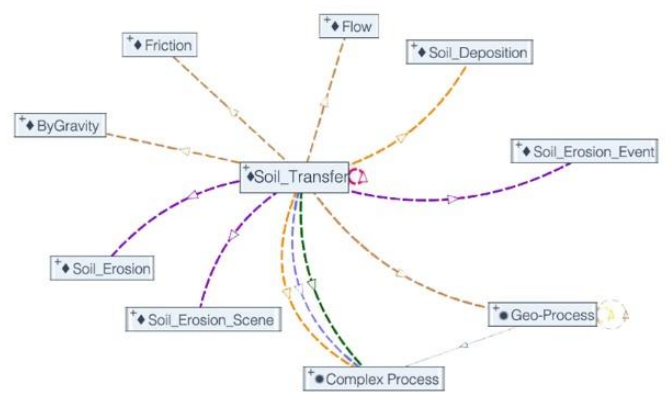

Figure 10: Soil Transfer Sub-Process Ontology Expression.

with a clear description of the geographic relations among elements, scenes and processes. Several questions related to the description of geographic processes can describe the evolution and mechanism of action of geographic processes, namely, when, where, what, how, and why. This paper describes geographic processes, geographic scenes, and geographic elements through the Time, Space, and Process ontologies and the relationship between the Spatiotemporal Relation ontology and the Element and Scene ontologies. This work also describes exactly how these geographic processes occur. Using the relationship

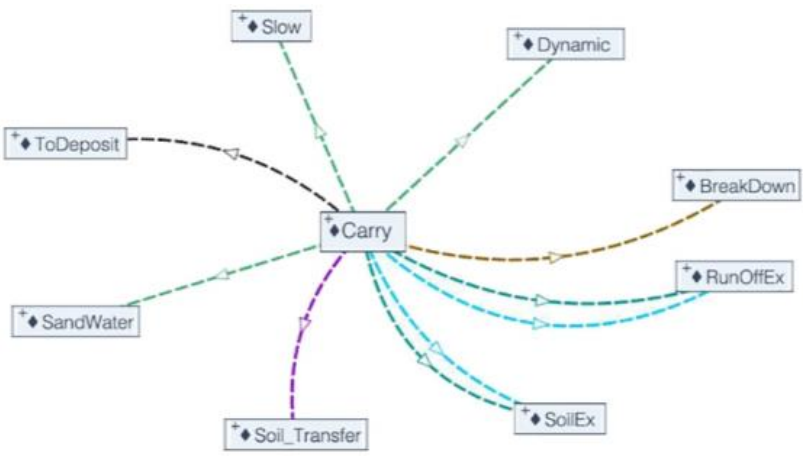

Figure 11: Carry action Ontology Expression.

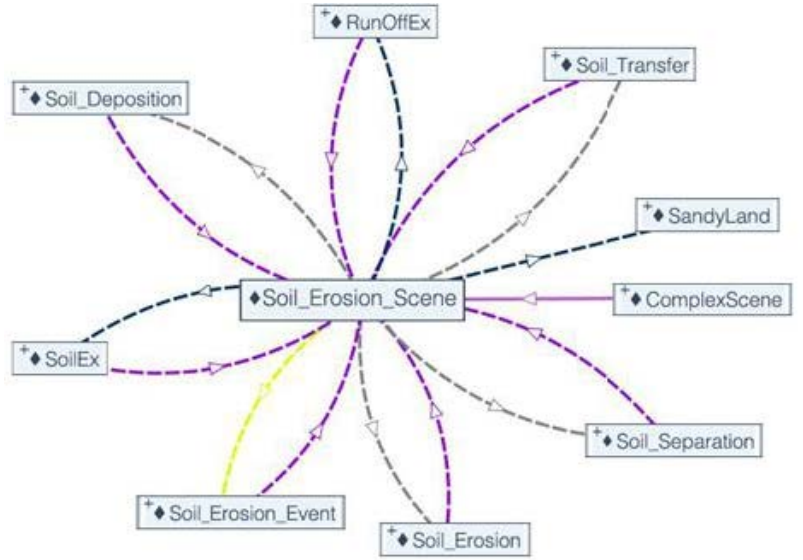

Figure 12: Erosion Scene Ontology Expression.

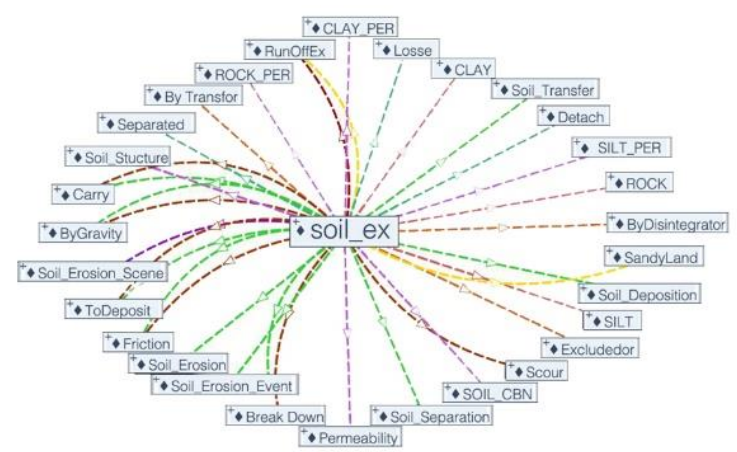

Figure 13: Soil Elements and Related Classes.

among the Element, Process, and Operation ontologies, this paper depicts the internal mechanisms of elements and processes while determining why they occurred. The geographic ontology model is suitable for how geographers interpret geographic phenomena. The Geographic Scene ontology proposed in this paper overcomes the lack of overall cognition in process-oriented models. The proposed ontology also focuses on the semantic and space-time relationships between ontologies and 
expresses the internal mechanism of the geographic process. This work establishes a network between several ontology models to clearly express the relationship between geographic entities and a full linkage of the connotations of the geographic process. This proposed framework completely expresses geographic processes through overall processes, sub-processes, elements, actions, and states along with the space-time relationship between the elements and the sub-processes.

Acknowledgments: This project was supported by the National Science Foundation of China (Grant No. 41631175).

Author Contributions: Yuwei Cao, Yi Huang and Yehua Sheng conceived and designed the experiments. Yuwei Cao performed the experiments and contributed reagents, materials and analysis tools.

Conflicts of Interest: The authors declare that they have no conflicts of interest.

\section{References}

[1] Blaut JM. Space and process. The professional geographer 1961;13:1-7.

[2] Goodchild MF. GIScience, geography, form, and process. Annals of the Association of American Geographers 2004;94:709-714.

[3] Wang S, Nakayama K, Kobayashi Y, Maekawa M. Considering events and processes within GIS: an event-based spatiotemporal data model. Communications and Information Technology, 2004 ISCIT 2004 IEEE International Symposium on; 2004: IEEE. p. 770-773.

[4] El-Geresy BA, Abdelmot AI, Jones CB. Spatio-temporal geographic information systems: a causal perspective. East European Conference on Advances in Databases and Information Systems; 2002: Springer. p. 191-203.

[5] Raper J, Livingstone D. Development of a geomorphological spatial model using object-oriented design. International Journal of Geographical Information Systems 1995;9:359-83.

[6] Grenon P, Smith B. SNAP and SPAN: Towards dynamic spatial ontology. Spatial cognition and computation 2004;4:69-104.

[7] Worboys M, Hornsby K. From Objects to Events: GEM, the Geospatial Event Model. Geographic Information Science, Third International Conference, GIScience 2004, Adelphi, MD, USA, October 20-23, 2004, Proceedings; 2004. p. 327-344.

[8] Galton A, Worboys M. Processes and events in dynamic geonetworks. International Conference on GeoSpatial Sematics; 2005: Springer. p. 45-59.

[9] Xue C, Zhou C, Fenzhen S, Dong Q, Xie J. Research on processoriented spatio-temporal data model. Acta Geodaetica et Cartographica Sinica 2010; 39(1): 95-101.

[10] Xie J, Xue C. A top-down hierarchical spatio-temporal process description method and its data organization. International
Symposium on Spatial Analysis, Spatial-Temporal Data Modeling, and Data Mining; 2009: International Society for Optics and Photonics. p. 74922P.

[11] Li R, Klippel A, Yang J. Geographic event conceptualization: where spatial and cognitive sciences meet. Proceedings of the Annual Meeting of the Cognitive Science Society; 2011.

[12] Studer R, Benjamins VR, Fensel D. Knowledge engineering: principles and methods. Data \& knowledge engineering 1998;25:161-197.

[13] Harding J. Geo-ontology concepts and issues. Ikley, UK: Ordance Survey 2002.

[14] Egenhofer MJ, Mark DM. Naive geography. International Conference on Spatial Information Theory; 1995: Springer. p. 1-15.

[15] Liu J, Li B, Shi L, Wang Z, Tao K. An automated retrieval method of geo-spatial event information based on ontology. Acta Geodaetica Et Cartographica Sinica 2011;40:502-508.

[16] Li B, Liu J, Shi L. Research on geo-ontology construction based on spatial affairs. International Conference on Earth Observation Data Processing and Analysis (ICEODPA); 2008: International Society for Optics and Photonics. p. 72852R.

[17] Smith B, Mark DM. Geographical categories: an ontological investigation. International journal of geographical information science 2001;15:591-612.

[18] LI W, YAN M, WANG J. Ontology-based data integration for intelligent transport systems. Journal of Central South University (Science and Technology) 2013;7:057.

[19] Buttigieg PL, Morrison N, Smith B, Mungall CJ, Lewis SE. The environment ontology: contextualising biological and biomedical entities. Journal of Biomedical Semantics 2013;4:1-9.

[20] Frew J., Freeston M., Freitas N., Hill L., Janée G., Lovette K. et al. The Alexandria Digital Library Architecture. International Journal on Digital Libraries 2000;2:259-268. https://link.springer. com/article/10.1007/PL00021470

[21] Raskin RG, Pan MJ. Knowledge representation in the semantic web for Earth and environmental terminology (SWEET). Computers \& geosciences 2005;31:1119-1125.

[22] de Lamos Dias T. T, Câmara G, Fonseca F, Davis C. Bottom-up development of process-based ontologies. Geographic Information Science: Third International Conference (GIScience 2004) Berlin, Springer Lecture Notes in Computer Science; 2004. p. 64-67.

[23] Frank AU. Ontology for spatio-temporal databases. SpatioTemporal Databases: Springer; 2003:9-77.

[24] Noy NF, Chugh A, Liu W, Musen MA. A framework for ontology evolution in collaborative environments. International semantic web conference; 2006: Springer. p. 544-558.

[25] Varanka DE. Ontology patterns for complex topographic feature types. Cartography and Geographic Information Science 2011;38:126-136.

[26] Gruber TR. A translation approach to portable ontology specifications. Knowledge acquisition 1993;5:199-220.

[27] Wu L., Xue L., Li C., Lv X., Chen Z., Jiang B. et al. A KnowledgeDriven Geospatially Enabled Framework for Geological Big Data. ISPRS International Journal of Geo-Information 2017;6:166.

[28] Wang C., Chen Z., Chen N., Wang W. A Hydrological Sensor Web Ontology Based on the SSN Ontology: A Case Study for a Flood. ISPRS International Journal of Geo-Information 2017;7:2.

[29] Fonseca FT. Ontology-driven geographic information systems. 2001. 
[30] Gal A, Modica G, Jamil H. Ontobuilder: Fully automatic extraction and consolidation of ontologies from web sources. Data Engineering, 2004 Proceedings 20th International Conference on; 2004: IEEE. p. 853.

[31] Uitermark HT, van Oosterom PJ, Mars NJ, Molenaar M. Ontologybased integration of topographic data sets. International Journal of Applied Earth Observation and Geoinformation 2005;7:97-106.

[32] Zhang C, Zhao T, Li W, Osleeb JP. Towards logic-based geospatial feature discovery and integration using web feature service and geospatial semantic web. International Journal of Geographical Information Science 2010;24:903-923.

[33] Castells P., Fernandez M., Vallet D. An Adaptation of the VectorSpace Model for Ontology-Based Information Retrieval. IEEE Transactions on Knowledge \& Data Engineering 2007;19:261272. https://ieeexplore.ieee.org/document/4039288
[34] Saruladha K, Aghila G, Raj S. A Survey of Semantic Similarity Methods for Ontology Based Information Retrieval. Second International Conference on Machine Learning and Computing; 2010. p. 297-301.

[35] Lü G, Chen M, Yuan L, et al. Geographic scenario: a possible foundation for further development of virtual geographic environments. International Journal of Digital Earth 2017:1-13.

[36] Claramunt $C$, Thériault $M$. Managing time in GIS an eventoriented approach. Recent advances in temporal databases: Springer; 1995:23-42.

[37] Yuan M. Representing complex geographic phenomena in GIS. Cartography and Geographic Information Science 2001;28:8396.

[38] García-Peñalvo F.J., de Pablos P., García J., Therón R. Using OWLVisMod through a decision-making process for reusing OWL ontologies. Behaviour \& Information Technology 2014;33:426442. 